\title{
Unspezifische klinische Symptomatik bei 2 Kleinkindern mit Spondylodiszitis
}

Die Spondylodiszitis im Kindesalter ist eine seltene Erkrankung, die sich durch sehr unterschiedliche und vor allem unspezifische Symptome manifestiert. Eine sichere Diagnose kann nur in der MRT (alternativ CT, falls MRT nicht möglich) gestellt werden. Wir berichten hier über 2 Patienten im Kleinkindalter mit dieser Erkrankung.

\section{Fallbericht Patient 1}

Die Erstvorstellung des Knaben in unserer Klinik erfolgte im Alter von 4 4/12 Jahren, da er seit etwa 4 Monaten an rezidivierenden Rückschmerzen litt. Diese traten zunächst episodenartig auf und hielten jeweils ca. 15 Minuten an. Die Schmerzen traten auch nachts auf, sodass der Patient davon erwachte. Zeitweise wurde eine Schonhaltung beobachtet. Schmerzbedingt war zuletzt der Kindergartenbesuch nur noch eingeschränkt möglich. Fieber wurde nicht beschrieben.

Es erfolgte zunächst eine kinderärztliche und kinderchirurgische Vorstellung, wobei eine Coxitis fugax und eine Pyelonephritis ausgeschlossen wurden. Im Anschluss daran stellte sich der Patient beim Osteopathen zum Ausschluss einer Blockade vor. Die kontinuierliche Gabe von Schmerzmitteln (Ibuprofen 3x tgl.) führte zu keiner wesentlichen Besserung der Symptomatik. Da die Familie kürzlich den Wohnort gewechselt hatte, wurde eine psychologische Vorstellung angeraten. Hier wurden Zeit und Ruhe zum Eingewöhnen in die neue Umgebung sowie Sport zur Steigerung des Körperbewusstseins empfohlen. Alle diese Maßnahmen brachten jedoch keine wesentliche Besserung. Die bisherige Eigenanamnese war unauffällig.

Bei der körperlichen Untersuchung bei uns wurden aktuell keine Schmerzen angegeben. Es bestand kein Druck- oder Klopfschmerz über der Wirbelsäule, keine lokale Rötung oder Schwellung und keine wesentliche Bewegungseinschränkung. So konnte z. B. das Knie problemlos an die Nase gezogen werden.

Ein Röntgenbild der Wirbelsäule war ebenfalls unauffällig. Daraufhin wurde eine MRT der Wirbelsäule veranlasst. Hier zeigte sich
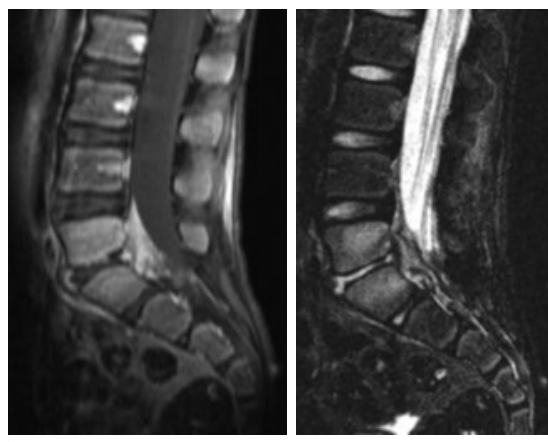

- Abb. 1 MRT bei Diagnosestellung von Patient 1.Spondylodiszitis mit geringer Diszitis LWK5/SWK 1 mit entzündlicher Beteiligung des Epiduralraumes sowie des Os sacrums, gering auch der prävertebralen Weichteile (links: fettgesättigte T1-Sequenz mit Kontrastmittel, rechts: fettgesättigte T2-Wichtung).

das Bild einer Spondylitis mit geringer Diszitis LWK 5/SWK 1 mit Betonung der linksseitigen Anteile und entzündlicher Beteiligung des Epiduralraumes ( $\triangleright$ Abb. 1). Bei den Laboruntersuchungen ergaben sich folgende erhöhte Parameter: BSG 68 mm/hr n.W., $\operatorname{lgA} 2,78 \mathrm{~g} / \mathrm{l}$; normwertig hingegen waren CrP, Procalcitonin, IgG, IgM, Differenzialblutbild sowie übliche Leber- und Nierenretentionsparameter. Die mikrobiologische Diagnostik umfasste einen Quantiferon-Test zum Ausschluss einer Tuberkulose, dreimalige Abnahmen einer Blutkultur (jeweils negativ) sowie verschiedene serologische Untersuchungen (Bartonellen, Brucellen), die alle unauffällig waren. Auf eine Biopsie wurde verzichtet.

Therapeutisch erfolgte eine kalkulierte antibiotische Behandlung mit Cefuroxim i.v. für 14 Tage, welche im Verlauf nach Abfallen der BSG auf $19 \mathrm{~mm} / \mathrm{hr} \mathrm{n}$. W. oral für weitere 4 Wochen fortgesetzt wurde. Nach 6 Wochen zeigte sich ein deutlich rückläufiger Befund in der MRT sowie ein Normalisieren der BSG. Seither ist der Patient seit etwa 2 Jahren völlig Beschwerde-frei.

\section{Fallbericht Patientin 2}

Das 14 Monate alte Mädchen wurde mit einer seit ca. 3 Wochen zunehmenden Gangstörung erstmals bei uns vorgestellt. Sie könne nicht mehr alleine frei laufen und würde sich auch nicht mehr selbstständig zum Stand hochziehen. Das Krabbeln sei nicht beeinträchtigt. Begonnen hätten die Beschwerden etwa 1 Woche nach einer Impfung (6-fach- und Prevenar $13^{\circledR}$-Impfung in den Oberschenkel). Kurz darauf sei die Patientin an einer fieberhaften Gastroenteritis mit Nachweis von Adenoviren erkrankt und wurde stationär in einem auswärtigen Krankenhaus behandelt. Dabei zeigten sich erstmals erhöhte Entzündungsparameter (CrP bis max. $25 \mathrm{mg} / \mathrm{l}$, BSG von 90 mm/hr n.W.). Nachdem sonografisch eine Coxitis ausgeschlossen wurde, erhielt die Patientin probatorisch für 5 Tage eine orale antibiotische Therapie mit Clindamycin und Ibuprofen als Bedarfsmedikation. Hierunter sei das Kind entfiebert, habe jedoch zur Behandlung vermeintlicher Schmerzen häufig Ibuprofen erhalten. Darüber hinaus bestand in den letzten 3 Wochen eine Infektion der oberen Atemwege. Zudem berichtete die Mutter, dass die Patientin sehr müde und abgeschlagen wirke. Die übrige Eigenanamnese war unauffällig.

Bei der klinischen Untersuchung zeigte sich keine Schonhaltung oder Schmerzhaftigkeit, das Krabbeln war regelrecht, jedoch ließ sich das Kind nicht zum Stehen ermutigen und Laufen wurde komplett verweigert.

Die Laboruntersuchungen zeigten eine deutlich erhöhte BSG mit 107 mm/hr n. W., ein mit 6,6 mg/l nur gering erhöhtes $\mathrm{CrP}$ sowie eine dezente Thrombozytose von $539000 / \mu l$. Alle anderen Parameter waren regelrecht.

Nachdem eine neurologische Abklärung ohne wegweisenden Befund erfolgt war, wurde schließlich eine MRT der Wirbelsäule durchgeführt, die eine Spondylodiszitis LWK 3/4 zeigte ( $\mathbf{A b b}$. 2). Es folgte eine CT-gesteuerte Biopsie, die aber ebenso wie die serologischen Untersuchungen und die wiederholte Blutkultur keinen Erregernachweis erbrachte. Auch eine Tuberkulose wurde mittels QuantiFERON-Test weitgehend ausgeschlossen.

Die Patientin erhielt Cefuroxim zunächst i. v. für 3 Wochen, da die BSG nur sehr langsam rückläufig war. Der Verlauf wurde durch eine Coronavirus-Infektion verkom- 


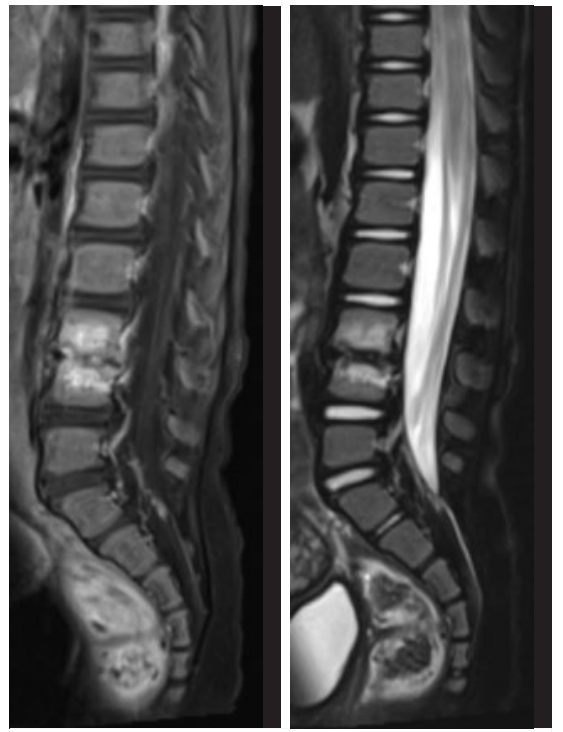

- Abb. 2 MRT bei Diagnosestellung von Patientin 2. Spondylodiszitis LWK3/ LWK4 mit paravertebraler und dorsal an der Wirbelkörperhinterkante gelegener entzündlicher Gewebevermehrung. (links: fettgesättigte T1-Sequenz mit Kontrastmittel, rechts: fettgesättigte T2-Wichtung).

pliziert. Anschließend wurde auf orale Gaben umgesetzt. Zudem erhielt die Patientin ein Stützkorsett. Es erfolgte eine MRT-Verlaufskontrolle 6 Wochen nach Diagnosestellung. Zu diesem Zeitpunkt war die Patientin beschwerdefrei und konnte wieder laufen. Hier zeigte sich jedoch eine fragliche Zunahme der Spondylodiszitis und auch die BSG war weiterhin mit $50 \mathrm{~mm} / \mathrm{hr}$ n. W. erhöht. Daher wurde die orale antibiotische Behandlung nochmals für 4 Wochen fortgesetzt und dann beendet. Seither geht es der Patientin seit nunmehr 6 Monaten gut. Das kürzlich durchgeführte MRT zeigte nun nur noch einen minimalen Restbefund.

\section{Diskussion}

Definitionsgemäß können Entzündungen der Wirbelsäule in eine Entzündung der Bandscheibe (Diszitis), des Wirbelkörpers (Spondylitis) oder eine Kombination aus beidem, der Spondylodiszitis, unterschieden werden. Bei Kindern liegt häufig eine Spondylodiszitis vor, da durch eine bessere Durchblutung des Anulus fibrosus der Bandscheibe sowie der Deckplatten der Wirbelkörper im Vergleich zu Erwachsenen die hämatogene Streuung der pathogenen Erreger begünstigt wird. In der westlichen
Welt wird die Inzidenz der Spondylodiszitis auf 1:250 000 geschätzt $[5,6,12]$ und macht etwa 1-3\% aller osteoartikulären Infektionen aus [12]. In Entwicklungsländern, wo sowohl Diagnostik als auch Therapie deutlich eingeschränkt sind und zudem andere Erreger, insbesondere Mykobakterien, eine wichtige Rolle spielen, mag die Inzidenz deutlich höher liegen.

Ätiologisch kann eine unspezifische, pyogene von einer spezifischen, durch Tuberkulose oder Brucellose bedingten, Spondylodiszitis unterschieden werden $[8,12,13]$. Sofern ein Erregernachweis der pyogenen Spondylodiszitis gelingt, wird bei Säuglingen bis zum 6. Lebensmonat in $80 \%$ der Fälle Staphylococcus aureus nachgewiesen [2, 3, 9]. In der Gruppe der Kleinkinder von 6 Monaten bis 4 Jahren, bei denen ein Erregernachweis generell schwierig ist, scheint hingegen eine Infektion mit Kingella kingae die häufigste Ursache zu sein [11]. Dieses gramnegative Bakterium ist in der Blutkultur nur schwer detektierbar. Ein Nachweis sollte im Biopsat mittels PCR versucht werden. Da Kingella kingae den Oropharynx besiedelt, kann außerdem versucht werden, den Erreger im Rachenabstrich nachzuweisen. So wurde in einer Studie bei Kindern mit osteoartikulären Infektionen im Alter von 6 Monaten bis 4 Jahren ohne Erregernachweis in der Blutkultur wiederholt im Rachenabstrich Kingella kingae nachgewiesen $[4,11]$. Dabei ist allerdings zu beachten, dass eine orale Besiedlung nur bedingt Rückschlüsse auf die Ätiologie der Spondylodiszitis zulässt. Andere Erreger der Spondylodiszitis im Kindesalter, die jedoch deutlich seltener nachgewiesen werden, stellen Staphylococcus epidermidis, Koagulase-negative Staphylokokken, alpha-hämolysierende Streptokokken, Streptococcus pneumoniae sowie andere Gram-negative Bakterien wie Escherichia coli und Salmonella spp. dar [12, 13].

Die Symptome der Spondylodiszitis hängen von der betroffenen Lokalisation ab und sind unspezifisch, wie auch bei den beiden hier vorgestellten Patienten. Am häufigsten ist die Lendenwirbelsäule betroffen, gefolgt von der Brust- und Halswirbelsäule. Es können Fieber, Rückenschmerzen, Schmerzen beim Wickeln, Verweigerung von Stehen und Laufen oder Tragen von Gegenständen sowie abdominale Beschwerden auf- treten. Neurologische Ausfälle sind selten und meistens durch eine tuberkulöse Spondylodiszitis bedingt [13]. Bei Befall der HWS kann es zu Nackenschmerzen oder Dysphagie kommen.

Durch die unspezifische Symptomatik kommt es häufig zu einer verzögerten Diagnosestellung der Spondylodiszitis. Labortechnisch sollten ein Differenzialblutbild, CrP sowie eine Blutkörperchensenkungsgeschwindigkeit (BSG) bestimmt werden. Die BSG ist meist erhöht, ebenso findet sich bei Diagnosestellung häufig eine moderate Erhöhung des CrP. Gelegentlich besteht eine leichte Leukozytose. Bei der zweiten hier vorgestellten Patientin fand sich hingegen eine leichte Thrombozytose. Bei akuten Verläufen kann auch die Bestimmung von Procalcitonin hilfreich sein. In der Regel ist aber der Wert bei der Spondylodiszitis bei Kindern im Unterschied zu erwachsenen Patienten nur selten erhöht [12].

Bei der bildgebenden Diagnostik stellt die konventionelle Röntgenaufnahme in der Regel die erste Maßnahme dar. Allerdings lassen sich dabei knöcherne Veränderungen erst nach einigen Wochen Krankheitsverlauf erkennen, sodass eine unauffällige Röntgenaufnahme der Wirbelsäule eine Spondylodiszitis nicht ausschließt. Bei unserem Patienten war ein auswärts durchgeführtes Röntgenbild der Wirbelsäule als unauffällig bewertet worden, obwohl die Beschwerden schon mehrere Wochen bestanden.

Daher stellt die MRT die Methode der Wahl zur Diagnose einer Spondylodiszitis dar. Hier zeigt sich üblicherweise ein Ödem im Wirbelkörper, das in der T1-Wichtung hypointens und in der T2-Wichtung und STIR-Sequenz hyperintens ist [12, 13]. Aufgrund der Strahlenbelastung sollte eine Computertomografie nur durchgeführt werden, falls eine MRT nicht möglich oder eine detaillierte Darstellung der knöchernen Verhältnisse der Wirbelkörper, insbesondere von Destruktionen, wichtig ist. Der Szintigrafie kommt bei der Diagnose der Spondylodiszitis keinerlei Bedeutung zu. Wird sie jedoch zur Suche nach infektiösen Herden bei unspezifischen Symptomen durchgeführt, so kann eine vermehrte Traceransammlung im entsprechenden Bereich auf eine Spondylodiszitis hinweisen. 
Es ist aber dann auf jeden Fall eine MRT zur Diagnosesicherung anzuschließen.

Ist die Diagnose einer Spondylodiszitis bestätigt, sollte immer ein Erregernachweis durch mehrmalige Blutkulturen versucht werden. Zudem muss eine Tuberkulose und gegebenenfalls auch eine Brucellose ausgeschlossen werden. Ob in jedem Fall eine Biopsie erfolgen soll, wird sehr kontrovers diskutiert. Völker und Kollegen vertreten die Meinung, dass aufgrund des erhöhten Risikos eines Eingriffes bei Säuglingen und Kleinkindern zunächst auf eine Intervention zum Keimnachweis verzichtet und eine kalkulierte Antibiotikagabe begonnen werden sollte [13]. Ähnlich sehen Principi und Esposito sowie Mohanty und Kollegen eine Indikation zur Nadelbiopsie nur bei Kindern, die nicht auf die antibiotische Therapie ansprechen, bei V.a. TBC oder zum Ausschluss einer malignen Erkrankung [10, 12].

Falls eine Biopsie veranlasst wird, sollte insbesondere bei Kleinkindern eine PCR-basierte Untersuchung auf Kingella kingae erfolgen. Leider war dies bei unserer Patientin mangels ausreichenden Materials nicht möglich. Alternativ kann ein Rachenabstrich auf Kingella kingae erwogen werden (siehe oben).

Therapeutisch kommt der antibiotischen Behandlung die größte Bedeutung zu. Allerdings gibt es für das Kindesalter, anders als im Erwachsenenalter, keine allgemein gültigen Empfehlungen zur Behandlung der Spondylodiszitis. Eine kalkulierte antibiotische Therapie muss in jedem Fall Staphylococcus aureus, bei kleinen Säuglingen zudem gramnegative Erreger abdecken. Bei Kleinkindern sollte das Antibiotikum auch Kingella kingae erfassen. Es scheint daher ähnlich den Empfehlungen für eine bakterielle Osteomyelitis [1] eine Behandlung mit Ampicillin/Sulbactam, Amoxicillin/ Clavulansäure oder Cefuroxim sinnvoll. Bei jungen Säuglingen sollte immer auch die Gabe eines Aminoglykosids dazu erwogen werden. Bei älteren Kindern ab dem 5. Lebensjahr kann alternativ auch Flucloxacilllin oder Clindamycin zum Einsatz kommen. Letztere haben allerdings eine ungenügende Wirksamkeit gegen Kingella kingae. Falls ein Erregernachweis gelingt, sollte im Verlauf eine entsprechende Anpassung der Antibiotika-Therapie erfolgen. Die optimale
Dauer der antibiotischen Behandlung ist nicht bekannt. Häufig wird eine mindestens 2-wöchige intravenöse Antibiose empfohlen, gefolgt von einer 4-8(-24)-wöchigen oralen Fortführung der Antibiose [10, $12,13]$ unter Kontrolle der Entzündungsparameter und der klinischen Symptomatik. Eine deutlich kürzere antibiotische Behandlung (3-7 Tage i. v., 20-30 Tage p.o.) bei unkompliziertem Verlauf wurde kürzlich von einer italienischen Gruppe postuliert [7]. Laut aktuellen Empfehlungen der Deutschen Gesellschaft für Infektiologie soll eine unkomplizierte Osteomyelitis 3 Wochen antibiotisch behandelt werden, bei Befall der Wirbelsäule muss die Behandlungsdauer jedoch individuell festgelegt und in der Regel länger verabreicht werden [1]. So haben wir unseren 1. Patienten 2 Wochen i. v. und 4 Wochen oral antibiotisch behandelt. Die 2. Patientin hingegen erhielt bei weiter erhöhter BSG und nicht eindeutigem Rückgang des Befundes in der MRT für 3 Wochen eine intravenöse und insgesamt 10 Wochen eine orale antibiotische Therapie.

Im Falle einer tuberkulösen Spondylodiszitis muss eine antituberkulöse Kombinationsbehandlung für ca. 9-12 Monate erfolgen. Eine spinale Brucellose wird antibiotisch mit Trimethoprim/Sulfamethoxazol und Rifampicin oder Gentamicin für 3-6 Monate behandelt. Bei älteren Kindern und Jugendlichen können auch Tetracycline verwendet werden.

Neben der antibiotischen Behandlung ist eine Ruhigstellung gegebenenfalls mittels Korsett notwendig. Eine chirurgische Intervention ist meist nur bei neurologischen Ausfällen, bei Abszessbildung oder ausgeprägten Destruktionen der Wirbelkörper notwendig.

Eine erste Verlaufskontrolle der Bildgebung ist bei unkomplizierten Verläufen der Spondylitis nach etwa 6 Wochen zur Beurteilung des Ansprechens auf die Therapie empfohlen. Eine weitere Verlaufskontrolle sollte einige Wochen nach Beendigung der antibiotischen Therapie erfolgen. Wir führten diese etwa 6 Monate nach Therapiebeginn durch.

Die Langzeitprognose der pyogenen Spondylodiszitis ist im Kindesalter insgesamt gut [10], so wie auch die beiden hier vorgestellten Patienten derzeit beschwerdefrei sind.

\section{FAZIT FÜR DIE PRAXIS}

Bei Rückenschmerzen oder motorischen Einschränkungen des Laufens oder Stehens im Kleinkindalter muss bei erhöhten Entzündungsparametern immer auch eine Spondylodiszitis differenzialdiagnostisch in Erwägung gezogen werden. Ein Erregernachweis sollte auf jeden Fall in der Blutkultur versucht werden. Inwieweit immer eine Biopsie empfehlenswert ist, bleibt offen. Die antibiotische Therapie sollte gerade bei Kindern im Alter von 6 Monaten bis 4 Jahren auch Kingella kingae erfassen.

Interessenkonflikt

Die korrespondierende Autorin gibt an, dass kein Interessenkonflikt besteht.

\section{Einhaltung ethischer Richtlinien}

Die korrespondierende Autorin bestätigt, dass für die Publikation dieses Fallberichts eine Einverständniserklärung der Eltern der Patienten vorliegt.

\section{Autorinnen}

Almut Meyer-Bahlburg', Roswitha Bruns ${ }^{1}$, Robin Bülow ${ }^{2}$, Sylke Otto ${ }^{2}$

${ }^{1}$ Klinik für Kinder- und Jugendmedizin, Universität Greifswald

2Diagnostische Radiologie, Universitätsmedizin Greifswald

\section{Korrespondenzadresse}

Prof. Dr. Almut Meyer-Bahlburg
Universitätsmedizin Greifswald
Klinik und Poliklinik für Kinder- und
Jugendmedizin
Ferdinand-Sauerbruch-Straße,
17474 Greifswald
Almut.Meyer-Bahlburg@med.uni-
greifswald.de


[1] Berner R, Bialek R, Forster ] et al, Hrsg. DGPI Handbuch. Infektionen bei Kindern und Jugendlichen. 7., vollständig überarbeitete Auflage. Stuttgart, New York: Georg Thieme; 2018

[2] Ceroni D, Kampouroglou G, Valaikaite R et al. Osteoarticular infections in young children: what has changed over the last years? Swiss medical weekly 2014; 144: w13971. doi:10.4414/smw.2014.13971

[3] Digby JM, Kersley JB. Pyogenic non-tuberculous spinal infection: an analysis of thirty cases. The Journal of bone and joint surgery. British volume 1979; 61 (1): 47-55

[4] El Houmami N, Minodier P, Dubourg G et al. Patterns of Kingella kingae Disease Outbreaks. The Pediatric infectious disease journal 2016; 35 (3): 340-346. doi:10.1097/INF.0000000000001010.

[5] Ferroni A, Al Khoury H, Dana C et al. Prospective survey of acute osteoarticular infections in a French paediatric orthopedic surgery unit. Clinical microbiology and infection 2013; 19 (9): 822-828. doi:10.1111/clm.12031

[6] Fucs PM de Moraes Barros, Meves R, Yamada HH. Spinal infections in children: a review. International orthopaedics 2012; 36 (2): 387-395. doi:10.1007/s00264-011 1388-2

[7] Giordano M, Aulisa AG, Guzzanti V et al. Managing of musculoskeletal infections in children. European review for medical and pharmacological sciences 2019; 23 (2 Suppl): 179-186. doi:10.26355/ eurrev_201904_17488

[8] Herren C, Jung N, Pishnamaz M et al. Spondylodiscitis: Diagnosis and Treatment Options. Deutsches Arzteblatt international 2017; 114 (51-52): 875-882. doi:10.3238/ arztebl.2017.0875

[9] Kayser R, Mahlfeld K, Greulich M et al. Spondylodiscitis in childhood: results of a long-term study. Spine 2005; 30 (3): 318-323

[10] Mohanty CB, Fieggen G, Deopujari Chandrashekhar E. Pediatric spinal infections - a review of non-tuberculous infections.
Child's nervous system : ChNS : official journal of the International Society for Pediatric Neurosurgery 2018; 34 (10): 1947-1956. doi:10.1007/s00381-018-3885-8

[11] Principi N, Esposito S. Kingella kingae infections in children. BMC infectious diseases 2015; 15: 260. doi:10.1186/s12879-0150986-9

[12] Principi N, Esposito S. Infectious Discitis and Spondylodiscitis in Children. International journal of molecular sciences 2016; 17 (4): 539. doi:10.3390/ijms17040539

[13] Völker A, Schubert S, Heyde C-E. Spondylodiszitis im Kindes- und Jugendalter. Der Orthopade 2016; 45 (6): 491-499. doi:10.1007/s00132-016-3273-6

\section{Bibliografie}

DOI https://doi.org/10.1055/a-0992-3005 arthritis + rheuma 2019; 39: 414-417 (c) Georg Thieme Verlag KG Stuttgart · New York ISSN 0176-5167 